\title{
Erstmaliges Vorhofflimmern: Medikamente oder Katheterablation?
}

\section{Patienten mit paroxysmalem Vorhof- flimmern, die bislang noch keine anti- arrhythmische Therapie erhielten, können konservativ ebenso gut behandelt werden wie mit Katheter- ablation.}

- In die Studie wurden Patienten unter 70 Jahren aufgenommen, die innerhalb der letzten sechs Monate mindestens zwei Episoden von symptomatischem paroxysmalem Vorhofflimmern für eine Dauer von weniger als sieben Tage hatten, ohne Antiarrhythmika geblieben waren und deren linker Vorhof nicht größer als $50 \mathrm{~mm}$ war. Nach Zufallskriterien erhielten 146 eine oder auch wiederholte Ablationen, 148 wurden konservativ mit Antiarrhythmika der Klasse I (200 mg Flecainid oder 600 mg Propafenon, evtl. zusätzlich Betablocker, Kalziumantagonisten oder Digoxin) oder bei Kontraindikationen mit Medikamenten der Klasse III (200 mg Amiodaron oder $160 \mathrm{mg}$ Sotalol) behandelt. Nach 3, 6, 12, 18 und 24 Monaten oder bei Symptomen von Vorhofflimmern wurde jeweils sieben Tage lang das EKG aufgezeichnet.

Die Dauer von Vorhofflimmern in den einzelnen und in allen EKGs war in beiden Gruppen nicht signifikant unterschiedlich. Nur bei der Aufzeichnung nach 24 Monaten war sie bei Patienten der Ablationsgruppe mit 9\% gegen 18\% signifikant kürzer. $\mathrm{Zu}$ diesem Zeitpunkt waren auch mehr Ablationspatienten ohne Vorhofflimmern (85 vs. 71\%, ohne Symptome 93 vs. $84 \%$ ). Andererseits mussten 13 Patienten der Ablationsgruppe mit Antiarrhythmika behandelt werden, während $54(30 \%)$ der Kontrollgruppe eine Ablation erhalten hatten. In der Ablationsgruppe gab es einen tödlichen Apoplex im Zusammenhang mit dem Eingriff und drei Fälle von Herztamponade.
- Nielsen JC et al.

Radiofrequency ablation as initial therapy in paroxysmal atrial fibrillation. New Engl. J. Med. 2012; 367: 1587-1595

\section{Kommentar}

Vielfach wird gefordert, dass man schon bei der ersten Episode eines paroxysmalen Vorhofflimmerns die Ablation einsetzen solle. Die Autoren interpretieren ihre Ergebnisse - Erfolgsraten und Nebenwirkungen - als Bestätigung der Leitlinienempfehlung, solche Patienten zunächst konservativ medikamentös zu behandeln. Beide Verfahren verhindern Vorhofflimmer-Rezidive. Es wird aber auch deutlich, dass auf lange Sicht, $d$. h. nach zwei Jahren, die Ablation dem Vorhofflimmern etwas besser vorbeugt ( 85 vs. 71\%) und dass ungefähr jeder dritte Patient schließlich doch abladiert werden muss.

H. HOLZGREVE —

\section{Softdrinks erhöhen das Prostata-Krebsrisiko}

\section{Die Nahrungs-Kohlenhydrate sollen in Relation zum Prostatakarzinom stehen.}

— Für eine prospektive Studie wurden von Wissenschaftlern der Universität Lund (Schweden), die Trink- und Essgewohnheiten von 8128 Männern im Alter von 45 bis 73 Jahren über einen Zeitraum von 15 Jahren beobachtet. Bei 817 Männern wurde in diesem Zeitraum ein Prostata-Ca. diagnostiziert.

Schon bei dem Genuss von einem Softdrink pro Tag stieg die Gefahr, an einer aggressiven Form des Prostatakrebses zu erkranken. Bereits 330 Milliliter am Tag reichten aus, um das Risiko um $38 \%$ zu steigern.

Bei Liebhabern zuckerhaltiger Frühstücksflocken stieg die Gefahr, an einer milderen Form des Prostatakrebses zu

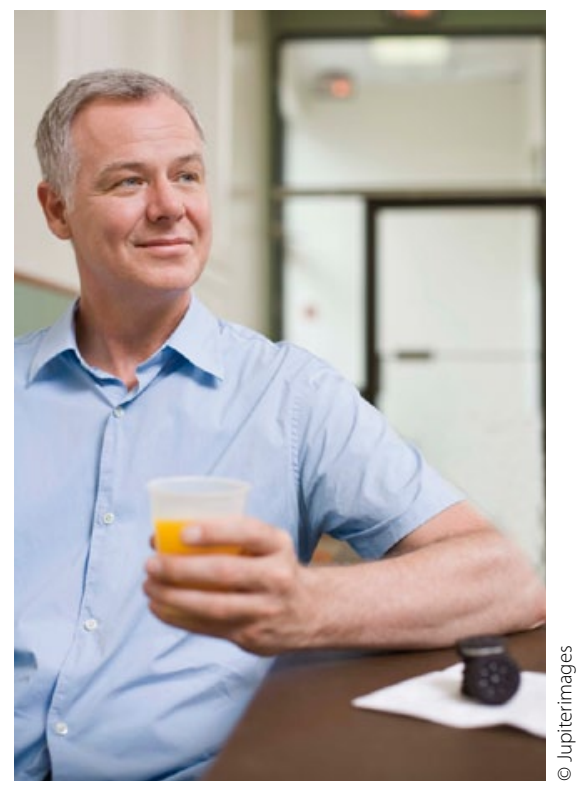

erkranken, die allerdings nicht behandelt werden muss.

\footnotetext{
- I. Drake et a.I

Dietary intakes of carbohydrates in relation to prostate cancer risk: a prospective study in the Malmö Diet and Cancer cohort. Am J Clin Nutr 2012 96: 6 1409-1418; First published online November 7, 2012. doi:10.3945/ ajcn.112.039438
}

\section{Kommentar}

Vor einer Änderung der Ernährungsrichtlinien sind noch weitere Untersuchungen notwendig. Doch schon jetzt gibt es für jeden Einzelnen genügend Gründe, seinen Limonadenkonsum zu beschränken.

K. MALBERG
Softdrinkliebhaber haben häufiger aggressive Prostatakarzinome. 